\title{
Imunoterapia como tratamento de câncer e o papel da enfermagem
}

\author{
Immunotherapy as cancer treatment and the role of nursing \\ La inmunoterapia como tratamiento del cáncer y el papel de la enfermería
}

Recebido: 08/05/2021 | Revisado: 12/05/2021 | Aceito: 16/05/2021 | Publicado: 07/06/2021

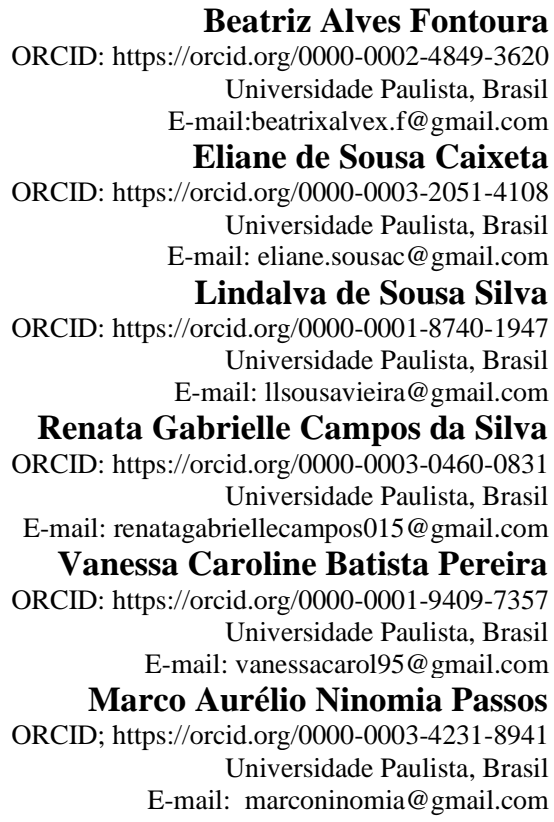

\section{Resumo}

Objetivo: O objetivo desse trabalho foi descrever as técnicas e aplicações da imunoterapia, como elas interagem com os tumores e as perspectivas e benefícios dessas novas terapias para os pacientes, apresentando as diversas formas de tratamento contra o câncer, identificando as consequências, verificando a produção cientifica a respeito da imunoterapia com as finalidades de demonstrar a eficácia para o tratamento do câncer, apresentando as vantagens das terapias utilizadas e os avanços nas descobertas de novos métodos terapêuticos, e apontando o papel da enfermagem na imunoterapia. Metodologia: Trata-se de revisão integrativa com análise descritiva, que tem sido considerada, hoje em dia, uma ferramenta muito importante na elaboração de estudos no campo da saúde. Resultados: Estão descritas informações gerais dos 11 artigos incluídos nesta revisão integrativa. Foram interpretados e sintetizados todos os resultados, através de uma comparação dos dados evidenciados na análise dos artigos ao referencial teórico. Conclusão: A imunoterapia é um tratamento contra o câncer em desenvolvimento. Há alguns pontos importantes a serem revisados sobre o papel da enfermagem no tratamento em diferentes tipos de tumores. Por causa de sua combinação ou da ordem de tratamento envolvida, eles são muito amplos e devem ter mais de uma via de proteção diferente dos tratamentos existentes e determinar uma programação ideal.

Palavras-chave: Imunoterapia; Câncer; Neoplasias; Enfermagem.

\begin{abstract}
Objective: The objective of this work was to describe the techniques and applications of immunotherapy, how they interact with tumors and the perspectives and benefits of these new therapies for patients, presenting the different forms of treatment against cancer, identifying the consequences, verifying the production scientific about immunotherapy with the purpose of demonstrating the efficacy for the treatment of cancer, presenting the advantages of the therapies used and the advances in the discoveries of new therapeutic methods, and pointing out the role of nursing in immunotherapy. Methodology: This is an integrative review with descriptive analysis, which has been considered, nowadays, a very important tool in the elaboration of studies in the health field. Results: General information on the 11 articles included in this integrative review is described. All results were interpreted and synthesized, by comparing the data shown in the analysis of the articles to the theoretical framework. Conclusion: Immunotherapy is a treatment for cancer in development. There are some important points to be reviewed about the role of nursing in the treatment of different types of tumors. Because of their combination or the order of treatment involved, they are very broad and must have more than one protection route different from existing treatments and determine an ideal schedule.
\end{abstract}

Keywords: Immunotherapy; Cancer; Neoplasms; Nursing. 


\begin{abstract}
Resumen
Objetivo: El objetivo de este trabajo fue describir las técnicas y aplicaciones de la inmunoterapia, cómo interactúan con los tumores y las perspectivas y beneficios de estas nuevas terapias para los pacientes, presentando las diferentes formas de tratamiento contra el cáncer, identificando las consecuencias, verificando la producción. científico sobre inmunoterapia con el propósito de demostrar la eficacia para el tratamiento del cáncer, presentar las ventajas de las terapias empleadas y los avances en los descubrimientos de nuevos métodos terapéuticos, y señalar el papel de la enfermería en la inmunoterapia. Metodología: Se trata de una revisión integradora con análisis descriptivo, que se ha considerado, en la actualidad, una herramienta muy importante en la elaboración de estudios en el campo de la salud. Resultados: Se describe información general sobre los 11 artículos incluidos en esta revisión integradora. Todos los resultados fueron interpretados y sintetizados, comparando los datos mostrados en el análisis de los artículos con el marco teórico. Conclusión: La inmunoterapia es un tratamiento para el cáncer en desarrollo. Hay algunos puntos importantes que deben revisarse sobre el papel de la enfermería en el tratamiento de diferentes tipos de tumores. Por su combinación o el orden de tratamiento involucrado, son muy amplios y deben tener más de una vía de protección diferente a los tratamientos existentes y determinar un esquema ideal.
\end{abstract}

Palabras clave: Inmunoterapia; Cáncer; Neoplasias; Enfermería.

\title{
1. Introdução
}

O câncer é uma doença com fator genético, mas também pode estar relacionado aos fatores ambientais e de estilo de vida (Borghaei, 2009).

O câncer é o principal problema de saúde pública no mundo e já está entre as quatro principais causas de morte prematura (antes 70 anos de idade) na maioria dos países. A incidência e a mortalidade por câncer vêm aumentando no mundo, em parte pelo envelhecimento, pelo crescimento populacional, como também pela mudança na distribuição e na prevalência dos fatores de risco de câncer, especialmente aos associados ao desenvolvimento socioeconômico. Verifica-se uma transição dos principais tipos de câncer observados nos países em desenvolvimento, com um declínio dos tipos de câncer associados a infecções e o aumento daqueles associados à melhoria das condições socioeconômicas com a incorporação de hábitos e atitudes associados à urbanização (sedentarismo, alimentação inadequada, entre outros) (Bray et al, 2018).

A vigilância de câncer, no escopo das ações de controle das doenças não transmissíveis, apoiada nas informações de morbimortalidade obtidas pelos Registros de Câncer de Base Populacional (RCBP), Registros Hospitalares de Câncer (RHC) e pelo Sistema de Informações sobre Mortalidade (SIM) do Departamento de Informática do Sistema Único de Saúde (DATASUS), fornece os subsídios para que os gestores monitorem e organizem as ações para o controle de câncer, bem como o direcionamento da pesquisa em câncer (Mathers et al, 2013).

Para o Brasil, a estimativa para cada ano do triênio 2020-2022 aponta que ocorrerão 625 mil casos novos de câncer (450 mil, excluindo os casos de câncer de pele não melanoma). O câncer de pele não melanoma será o mais incidente (177 mil), seguido pelos cânceres de mama e próstata (66 mil cada), cólon e reto (41 mil), pulmão (30 mil) e estômago (21 mil). O cálculo global corrigido para o sub-registro, aponta a ocorrência de 685 mil casos novos (Mathers et al, 2013).

Por diversos anos, a luta contra o câncer foi baseada de acordo com esse tripé: cirurgia, quimioterapia e radioterapia. Embora o uso dessa estratégia tenha alcançado um sucesso crescente, especialmente em tumores que foram descobertos anteriormente, a recorrência do tumor e/ou a progressão da doença se tornaram um problema de longo prazo no tratamento de pacientes com câncer (Mittal et al, 2014).

De acordo com a resposta imune pode ser dividida em fluidos corporais e células. Em primeiro lugar, os anticorpos produzidos pelos linfócitos B eliminam os agentes externos e toxinas; a reação do tipo celular elimina os agentes prejudiciais ao reconhecer os antígenos, ativar as células apresentadoras de antígenos e, finalmente, ativar e proliferar as células T. O local da imunoterapia atualmente, o câncer é uma das doenças com maior mortalidade do mundo. Os tumores podem ser classificados como benignos ou malignos, a imunoterapia pode ser usada nos dois tipos de tumores, porém são mais utilizados em malignos podem metastatizar (Antonia, 2014). 
$\mathrm{Na}$ última década, muitos estudos conseguiram explicar o importante papel do sistema imunológico no combate e eliminação das células tumorais, mas é importante observar que o próprio sistema imunológico também pode desempenhar um papel no desenvolvimento de tumores (Silva, 2003).

O processo de supressão do sistema imunológico e/ou promoção do crescimento tumoral é denominado imunoedição e pode ser dividido em três etapas ${ }^{4}$. Na fase de eliminação, uma característica é pela ativação da resposta imune inata e, em última instância, as células tumorais são destruídas por um sistema imune eficaz. Dependendo do estágio de equilíbrio, algumas células tumorais podem sobreviver ao ataque do sistema imunológico. Modifique as células sobreviventes. Conforme a fase de escape, as células tumorais modificadas e editadas começam a proliferar e estabelecer um ambiente tumoral imunossupressor (Parkin, 2010).

O sistema imunológico é o principal meio de tratamento dessa doença e oferece uma nova perspectiva para o tratamento do câncer. Este novo método alternativo é chamado de imunoterapia. Há extensas pesquisas sobre como o sistema imunológico atua as células tumorais. Esta tecnologia pode usar receptores de membrana celular, células linfoides, códigos genéticos para certas células de defesa e vacinas contra genes tumorais específicos (Hanahan, 2000).

A imunoterapia é um tipo de tratamento biológico que tem o objetivo de potencializar o sistema imunológico de maneira a que este possa combater infecções e outras doenças como o câncer. Nas últimas décadas, a imunoterapia tornou-se uma parte importante do tratamento de alguns tipos de câncer. Novos tipos de tratamentos imunoterápicos estão sendo estudados e terão grande impacto no tratamento do câncer. A imunoterapia inclui tratamentos que agem de diferentes formas. Alguns estimulam o sistema imunológico do corpo de uma forma muito geral, enquanto outros ajudam o sistema imunológico a atacar especificamente as células cancerígenas. A imunoterapia vem se mostrando mais eficaz em alguns tipos de câncer, como melanoma, câncer renal, câncer de pulmão, só para citar alguns. Nos últimos dois anos vem se mostrando que a associação da imunoterapia com quimioterapia tradicional ou com outras terapias alvo associadas pode ser ainda mais eficaz que a imunoterapia isolada (Oncologia, 2021).

Nos últimos anos, o uso da imunoterapia no tratamento do câncer tem se tornado uma estratégia atraente e interessante, principalmente porque pode ser utilizada no tratamento de diversos tumores. Esta terapia é baseada em uma maneira simples de usar o próprio sistema imunológico para induzir uma resposta antitumoral (Borghaei et al, 2009).

O progresso da pesquisa neste campo é surpreendente e a uma velocidade incrível. Novas terapias surgem há cada vez mais novos sinais e resultados a cada momento positivos (Bray et al, 2018; Mathers et al, 2013). Podemos mencionar alguns desses tratamentos aqui: vacinas anticâncer a imunoterapia com vírus oncolíticos é uma modadlidade de tratamento, ainda em estudos, onde vírus modificados são utilizados para combater determinados tipos de câncer (Yaddanapudi et al, 2010).

As células T usadas na terapia com células T CAR são alteradas em laboratório identificando as células cancerígenas específicas adicionando um receptor artificial, denominada receptores de antígeno quimérico ou CAR, o que permite a identificação dos antígenos específicos das células cancerígenas. Como diferentes tipos de câncer têm diferentes antígenos, cada CAR é feito para um antígeno específico. Por exemplo, determinados tipos de leucemia ou linfoma têm um antígeno denominado CD-19. Para tratar esses cânceres, a terapia com células T CAR são produzidas para se conectar ao antígeno CD-19 e não funcionarão para outro tipo de câncer que não tenha esse antígeno. As próprias células $\mathrm{T}$ do paciente são usadas para produzir as células T do CAR. Além de sua alta complexidade e custo, a realização desta modalidade de tratamento exige imunossupressão do paciente antes de sua aplicação, com o objetivo de melhorar sua eficácia. Uma alternativa de fonte de linfócitos é o sangue periférico, estratégias para isolar e expandir estas raras células T. específicas estão sendo desenvolvidas para estudos clínicos em populações maiores Os Linfócitos presentes em tumores transplantáveis são capazes de reconhecer células tumorais in vitro, onde são clonalmente expandidos e reinfundidos para o tratamento, porém a técnica de isolar e expandir clonalmente estes linfócitos é muito cara e trabalhosa, o que limita seu uso (Restifo \& Rosenberg, 2015) 
No início dos anos 2000 foram introduzidos linfócitos modificados geneticamente. Para aumentar sua especificidade, foram desenvolvidos linfócitos T com receptores antigênicos quiméricos (CAR T cells), que reconhecem antígenos tumorais sem a necessidade de apresentação pelas APCs (Antonia et al, 2014; Borghaei et al, 2009; Parkin, 2010).

O uso da imunoterapia para tratar o câncer é uma abordagem em desenvolvimento, e há alguns pontos importantes a serem revisados sobre o papel potencial do tratamento em diferentes tipos de tumores. A combinação ou sequência dessas terapias é muito ampla, devendo ter múltiplas vias de proteção diferentes das terapias existentes, e determinar o cronograma ideal (Couzin-Frankel, 2013). Também deve ser usado em combinação com imunoterapia na dose correta e combinado com radioterapia, quimioterapia ou medicamentos com outros mecanismos de ação. A vantagem do meio científico é que o conhecimento no campo da imunoterapia pode ser ampliado, sendo o uso dessa terapia pelo paciente o efeito mais específico que ela traz, podendo reduzir a terapia mais ativa para as células.

A prática de enfermagem precisa ser baseada em conceitos e pensamentos científicos para promover o progresso da teoria e da prática profissional, para que o conhecimento possa melhorar a eficiência do trabalho. No entanto, nem todos os enfermeiros com experiência em enfermagem requerem treinamento especializado. Nesse sentido, é importante ressaltar que para alcançar a plena eficiência no trabalho, educação e trabalho devem estar combinados para estimular o pensamento crítico, orientando-o a acumular e reconstruir conhecimentos no seu dia a dia profissional.

O objetivo desse trabalho foi descrever as técnicas e aplicações da imunoterapia, como elas interagem com os tumores e as perspectivas e benefícios dessas novas terapias para os pacientes, apresentando as diversas formas de tratamento contra o câncer, identificando as consequências, verificando a produção cientifica a respeito da imunoterapia com as finalidades de demonstrar a eficácia para o tratamento do câncer, apresentando as vantagens das terapias utilizadas e os avanços nas descobertas de novos métodos terapêuticos, e apontando papel da enfermagem na imunoterapia.

\section{Métodos}

Este trabalho trata-se de uma pesquisa de caráter qualitativo que segundo Knechtel (2014), a pesquisa busca entender fenômenos humanos por meio de uma análise cientifica do pesquisador. Com base em uma pesquisa de revisão bibliográfica cuja finalidade é proporcionar acesso à literatura produzida sobre determinado assunto, uma ferramenta muito importante na elaboração de estudos no campo da saúde, pois reúne, de forma sucinta, as pesquisas disponíveis sobre determinado assunto, direcionando o pesquisador na incorporação de evidências, o que promove a disseminação do conhecimento cientifico, permitindo aos profissionais das diversas áreas da saúde, acesso rápido aos resultados mais importantes de pesquisas, para a prática fundamentada no saber crítico (Mendes et al, 2008; Souza et al, 2010).

E foi elaborada a partir de material já publicado, de vários autores da área tais como Borghaei, (2009), relata que o câncer não é só um fato genético, Bray et al (2008) explica que o câncer é o principal problema de saúde pública, (Silva,2003) fala sobre o principal papel do sistema imunológico, conforme Oncologia (2021) a imunoterapia é um tratamento biológico com objetivo de potencializar o sistema imunológico, também se fez presente Instituto Nacional de Câncer (2015), que relata os preços dos medicamentos se tornem mais razoáveis no futuro próximo, e a avaliação das tecnologias em saúde no SUS, entre outros que contribuíram para esse trabalho.

Para determinar quais estudos serão incluídos neste estudo, os métodos utilizados para identificar as questões relevantes e as informações extraídas de cada estudo selecionado, iniciou-se o processo de definição das questões norteadoras, considerada a etapa mais importante do estudo (Couzin-Frankel, 2013). Para tanto, foi posteriormente desenvolvida uma ampla revisão da literatura, na qual a primeira etapa do processo teve início com a definição e seleção da hipótese de definição do tema. Nessa fase obteve-se a seguinte pergunta norteadora: Quais os principais métodos da imunoterapia? Inclui o uso eficaz do sistema imunológico para tratar o câncer? 
Para a obtenção dos artigos, foi realizado um levantamento em bancos de dados eletrônicos da Biblioteca Virtual em Saúde (BVS) - BIREME, Scientific Electronic Library Online - Scielo, Pubmed e Lilacs, além de lista de referências dos artigos identificados. A busca foi realizada a partir dos descritores: "Imunoterapia”, “câncer”, "neoplasias", "Brasil”, e "enfermagem”, entre o período de 2015 a 2020 e apenas artigos na língua portuguesa. A seleção dos descritores utilizados no processo de revisão foi efetuada mediante consulta ao DECs (descritores de assunto em ciências da saúde da BIREME).

Para responder a pergunta norteadora, foram adotados critérios de inclusão, sendo considerados aqueles artigos cujo acesso ao periódico era livre aos textos completos, artigos em idioma português, publicados e indexados nos últimos sete anos (2015 a 2020), que foram localizados através da busca com os seguintes descritores: "Imunoterapia”, “câncer”, "neoplasias”, "Brasil", e "enfermagem", que estavam relacionados à temática das "principais medidas a imunoterapia inclui o uso eficaz do sistema imunológico para tratar o câncer? Mas para isso é necessário criar um método que possa evitar que todas as células tumorais escapem, para que o tratamento seja bem-sucedido.

Como critérios de exclusão, estão artigos publicados em anos anteriores a 2015, em idiomas que não o português, que não apresentam relação com o tema proposto e a pergunta norteadora, além de que, optou-se por não incluir teses, dissertações e monografias, visto que a realização de uma busca sistemática das mesmas é inviável logisticamente.

Com os parâmetros utilizados, foram encontrados 582 artigos no banco de dados da Bireme e 352 artigos no banco de dados da Scielo, totalizando 125 artigos; para os resultados de cada busca, a seleção inicial ocorreu pela simples leitura dos títulos encontrados, sendo descartados aqueles evidentemente não relacionados ao tema; idioma em português; bem como o ano de publicação. Para os potencialmente elegíveis, os resumos foram avaliados para uma segunda etapa de seleção quanto à elegibilidade. Os artigos que aparentemente cumpriam com os critérios de inclusão, neste caso, 151 artigos foram obtidos e analisados na íntegra. Após a leitura criteriosa, apenas 11 artigos, atenderam rigorosamente aos critérios de inclusão.

A Figura 1 mostra o fluxograma da estratégia adotada para busca e inclusão dos artigos e as razões de exclusão de textos não inseridos.

Para extrair os dados relevantes de artigos selecionados, ferramentas previamente elaboradas com cuidado são usadas para coletar e sintetizar informações importantes, minimizando assim o risco de erros de transcrição e garantindo a precisão das informações usadas como registros (Couzin-Frankel, 2013).

Portanto, uma tabela é usada como uma ferramenta de mesclagem na qual as seguintes informações são agrupadas: a fim de melhor visualizar o número do artigo do artigo na leitura do conteúdo da discussão, título do artigo, autor(es), objetivo (s), método (s), conclusão e ano de publicação. 
Figura 1: Fluxograma detalhado do método aplicado na seleção dos artigos.

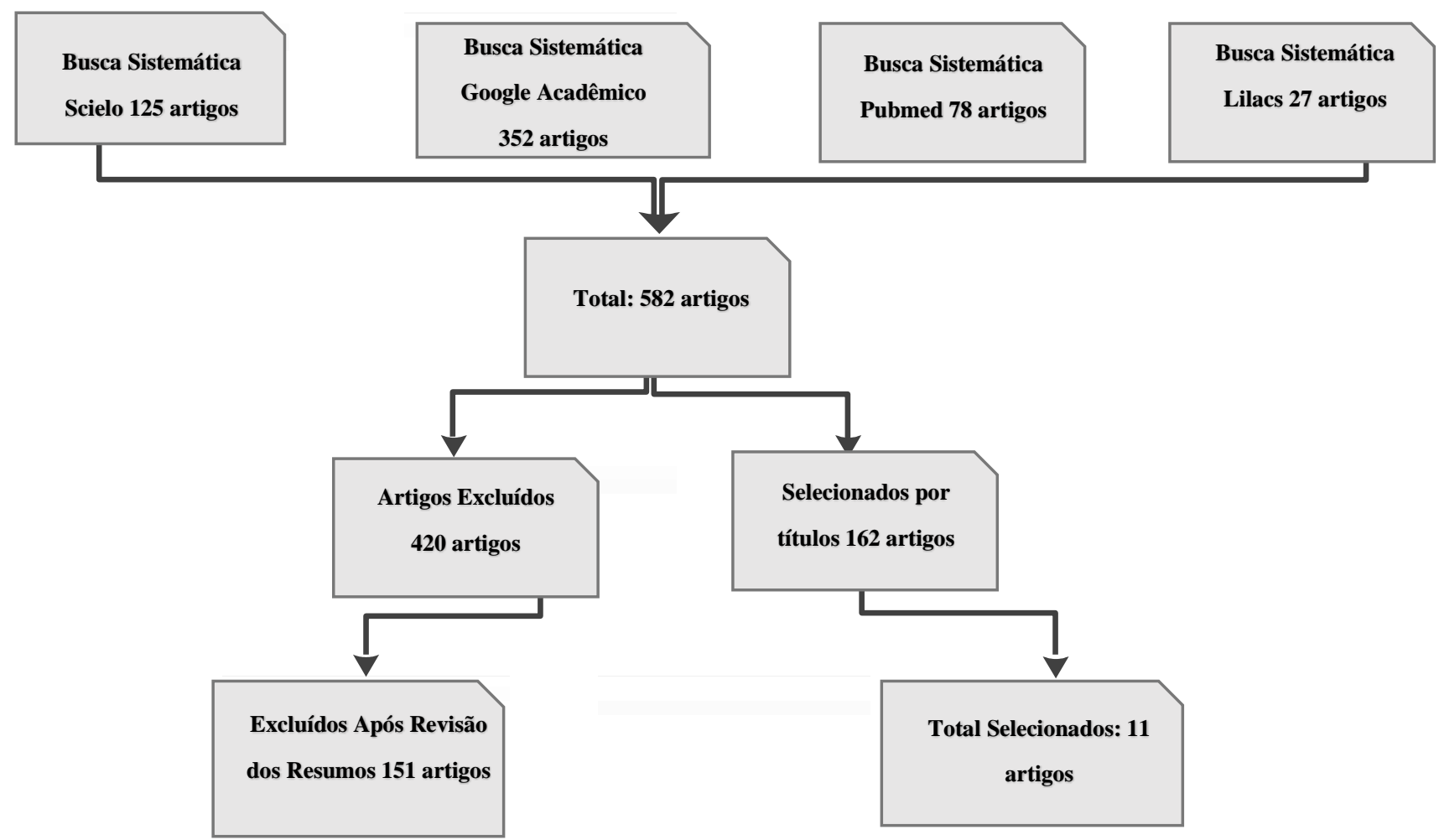

Fonte: Autores (2021).

\section{Resultados e Discussão}

\section{Descrição geral dos artigos selecionados}

Na Tabela 1 estão descritas informações gerais dos 11 artigos incluídos nesta revisão integrativa. Foram interpretados e sintetizados todos os resultados, através de uma comparação dos dados evidenciados na análise dos artigos ao referencial teórico (Souza et al, 2010).

Tabela 1 - Distribuição dos artigos de acordo com o título, autores, objetivo, método, conclusão e ano de publicação.

\begin{tabular}{|c|c|c|c|c|c|c|}
\hline & Título & Autor & Objetivo & Método & Conclusão & Ano \\
\hline $\overrightarrow{0}$ & $\begin{array}{l}\text { Análise do intervalo de tempo } \\
\text { entre o primeiro sintoma e o } \\
\text { Diagnóstico de mulheres com } \\
\text { câncer de mama em um centro } \\
\text { Oncológico de referência no } \\
\text { Rio deJaneiro }\end{array}$ & Medeiros GC. & \begin{tabular}{l} 
Avaliar os tempos entre o início \\
dos sintomas e a primeira \\
consulta médica (atras o pela \\
mulher) e entre a primeira \\
consulta médica e a confirmação \\
diagnóstica em laudo \\
histopatológico (atraso de \\
encaminhamento) em mulheres \\
com câncer de mama \\
matriculadas \\
\multicolumn{4}{c}{ no HCIII/INCA. }
\end{tabular} & $\begin{array}{l}\text { Estudo prospectivo em } \\
\text { uma coorte } \\
\text { de mulheres matriculadas } \\
\text { no Hospital do Câncer III / } \\
\text { Instituto Nacional de } \\
\text { Câncer } \\
\text { (HCIII/INCA). As } \\
\text { pacientes foram } \\
\text { entrevistadas entre } \\
\text { outubro de 2014 e abril de } \\
\text { 2015, no momento da } \\
\text { consulta de triagem, } \\
\text { utilizando questionário } \\
\text { semiestruturado. }\end{array}$ & $\begin{array}{l}\text { As principais barreiras que } \\
\text { contribuem para o atraso } \\
\text { em ambos os intervalos de tempo } \\
\text { são relacionadas a falhas no } \\
\text { sistema de saúde, na assistência e } \\
\text { no acesso à informação sobre o } \\
\text { CM. A identificação das } \\
\text { mulheres mais susceptíveis ao } \\
\text { atraso e as barreiras encontradas } \\
\text { durante o processo de } \\
\text { investigação do câncer poderá } \\
\text { resultar na elaboração de } \\
\text { propostas que visem o } \\
\text { diagnóstico precoce do CM }\end{array}$ & 2018 \\
\hline
\end{tabular}


Viabilidadesocioeconômica do Santos CVJ uso de imunoterapia no tratamento de câncer de pulmão.

Principais avanços no Lopes JD tratamento do melanoma cutâneo: imunoterapia e terapia alvo.

O uso do alphaherpesvírus Souza J, humano 1 oncolítico como Pauline C. estratégia terapêutica no controle de células metastáticas do câncer de mama.

O mercado brasileiro de Vidal TJ, anticorpos monoclonais Figueiredo TA, utilizados para o tratamento de Pepe VLE. câncer

Imunoterapia

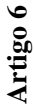

Silvestrini AA, Santos LHP dos.
Identificar as principais Trata-se de um estudo características dos anticorpos descritivo no ano 2016. monoclonais, destinados ao tratamento de câncer, com registro sanitário ativo, no Brasil, em 2016.
Este estudo analisa a questão da Foram entrevistados 8 O câncer é um dos maiores viabilidade socioeconômica do pessoas no total, sendo 4 desafios da humanidade. Vem umas das neoplasias com maior farmacêuticos menor escala ao longo dos incidência e mais alta taxa de e 2 administradores séculos e, apesar de todo mortalidade, através de hospitalares. As progresso da sociedade ao longo imunoterapia. instituições de atuação do tempo,

desse profissionais. permanece como obstáculo a ser vencido.

Descrever os métodos atualmente Trata-se de uma revisão de Mesmo a cirurgia de excisão 2018 utilizados para o tratamento do literatura. câncer de pele do tipo melanoma, com destaque para seus efeitos colaterais, a eficácia do tratamento, sendo observado que várias abordagens promissoras surgiram recentemente com a descoberta do mecanismo que levam às células.

Descrever o potencial oncolítico Trata-se de uma revisão de O tumor de mama é a principal do alphaherpesvírus humano 1 na literatura causa de morte por câncer em viroterapia de células metastáticas mamárias. mulheres e apresenta um sério desafio terapêutico em todo o mundo; constituindo a neoplasia maligna mais cara de se tratar. Sendo assim, o câncer mamário impõe um fardo aos pacientes e suas famílias, bem

como aos sistemas de saúde em todo o mundo.

Nesse sentido, este estudo apontou a necessidade de que se pense até que ponto as inovações propostas a esses novos produtos biotecnológicos são, de fato, inovadoras, para que não aconteça no mercado biotecnológico o mesmo que aconteceu com o mercado farmacêutico: o surgimento de um grande número de medicamentos método.

Descrever a importância do Trata-se de uma revisão de Nos últimos anos a utilização de sistema imunológico no combate literatura e eliminação de células tumorais, mas há que se ressaltar que o próprio sistema imunológico também pode ter atividade no processo de progressão tumoral. imunoterapia no tratamento 2016 oncológico tem se tornado uma estratégia atrativa e interessante principalmente pelo fato de poder ser utilizado contra um grande número de diferentes tumores.

Efeitos adversos da Faria ET, imunoterapia $\quad$ Diniz PER,

Gomes IP, Lima JF.

Efeitos adversos imunoterapia: lesões cutâneas e bucais

da Melo NS,

Mesquita CRM, Reis PED.
Nesse contexto, o diagnóstico Trata-se de uma revisão de precoce e o monitoramento literatura

clínico rigoroso são essenciais para o sucesso do manejo dos efeitos adversos da imunoterapia. Embora o tempo de início e a duração sejam bastante variados, de acordo com cada agente, a grande maioria dos efeitos adversos da imunoterapia reversível.

A imunoterapia está a ponto de Trata-se de uma revisão de transformar a prática da literatura oncologia, mas é de se esperar que, pela natureza de sua toxicidade, promoverá efeitos adversos novos ou modificará os já conhecidos.
A imunoterapia baseada em ICIs tem mudado paradigmas no tratamento de diversos cânceres. Apesar da introdução recente na prática clínica, a presença crescente dessa estratégia terapêutica torna fundamental a capacitação dos profissionais de saúde para o manejo de suas toxicidades.

Um exame sistemático da mucosa oral é recomendado como parte do regime de monitoramento de pacientes tratados com imunoterapia. $\mathrm{O}$ reconhecimento precoce e a pronta administração de cuidados terapêuticos podem limitar as modificações da dose, impedir a interrupção do tratamento, e, muito importante, preservar a qualidade de vida 


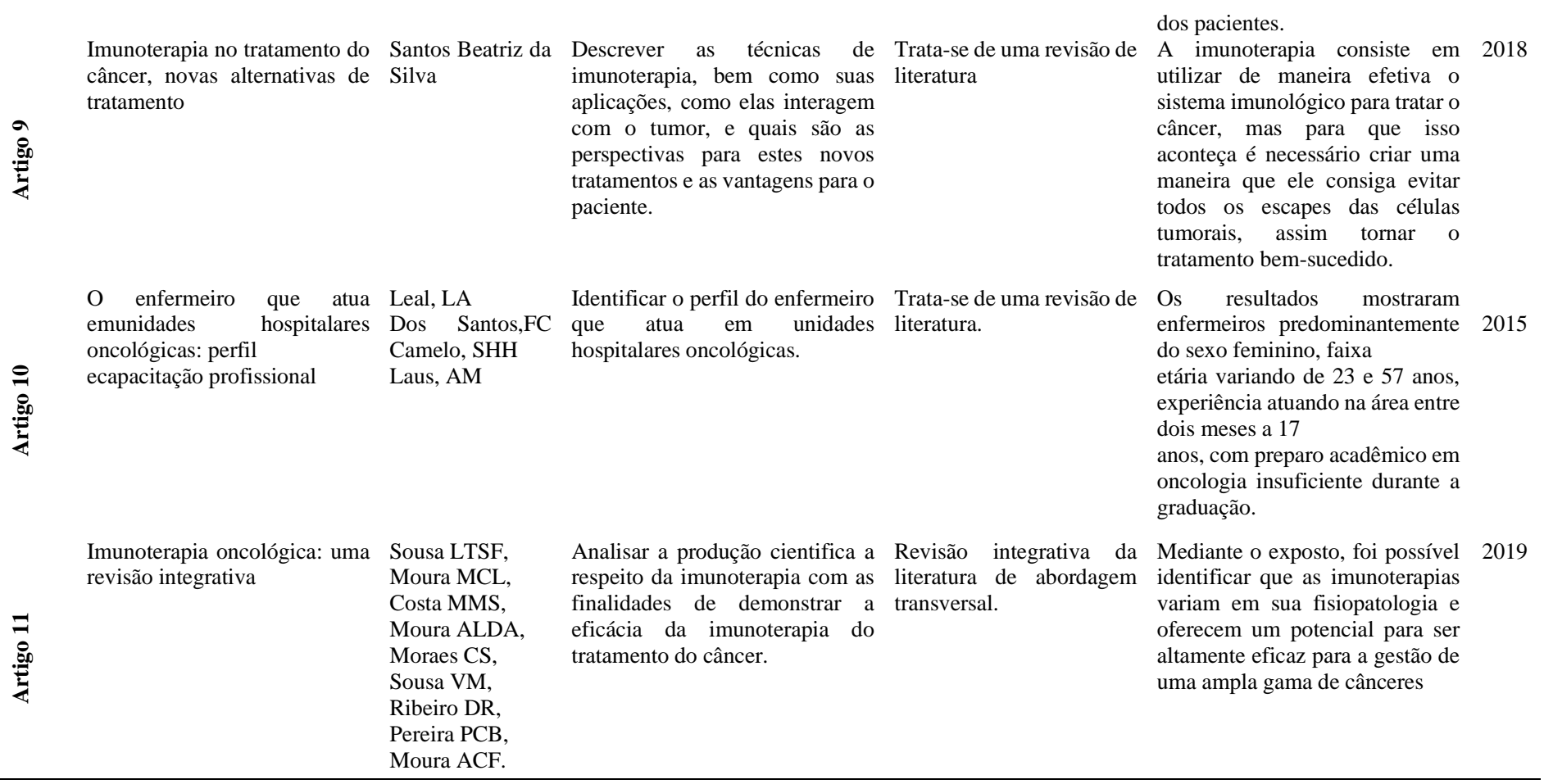

Fonte: Autores (2021).

Dentre os artigos incluídos nesta revisão integrativa, cinco (50\%) são de autoria de enfermeiros, dois (20\%) foi de autoria de médicos e enfermeiros e, em dois (30\%) outros profissionais da área da saúde em um (10\%) dos estudos, não foi possível identificar a categoria profissional de seus autores. Observou-se que, o grande número de publicações redigidas por enfermeiros, o que define claramente que esse é um tema muito abordado entre os profissionais de enfermagem e que estes devem estar intimamente ligados no processo do tratamento de câncer. Não houve predominância de veículo de publicação, sendo que os artigos foram publicados por várias revistas da área da saúde e os estudos realizados em diferentes estados, o que ressalta que a imunoterapia consolida-se como um tratamento menos agressivo e mais específico para cada tipo de câncer e paciente oncológico que os profissionais de saúde, tem interesse no que diz respeito ao tratamento do câncer.

A análise da pesquisa permite verificar a importância da capacitação e a necessidade de atualização em oncologia, pois dados da literatura mostram que o enfermeiro não está preparado para atuar na assistência ao paciente nesta área e é considerado um setor crítico (Souza et al, Amador 2010).

Com base na análise de conteúdo, foi possível identificar os estudos de acordo com os aspectos abordados sobre a percepção do câncer e categorizá-los em "Imunoterapia", "Câncer", "Neoplasias" e "Enfermagem". A maioria dos artigos abordou mais de uma categoria, sendo que, seis ( $80 \%$ ) deles discorreram sobre os benefícios da imunoterapia; dez (10\%) sobre o câncer; cinco (50\%) sobre neoplasias e um (1\%) relata sobre a atuação do enfermeiro na imunoterapia. As categorias nas quais cada artigo foi incluído podem ser vistas no Quadro 1, conforme disposição dos artigos na Tabela 1. 
Quadro 1 - Distribuição dos artigos de acordo com a categoria temática.

\begin{tabular}{|c|c|c|c|c|c|c|c|c|c|c|c|}
\hline Número do Artigo & 1 & 2 & 3 & 4 & 5 & 6 & 7 & 8 & 9 & 10 & 11 \\
\hline Imunoterapia & - & $\mathrm{x}$ & $\mathrm{x}$ & - & - & $\mathrm{x}$ & $\mathrm{x}$ & $\mathrm{x}$ & $\mathrm{x}$ & $\mathrm{x}$ & $\mathrm{x}$ \\
\hline Câncer & $\mathrm{x}$ & $\mathrm{x}$ & - & $\mathrm{x}$ & $\mathrm{x}$ & $\mathrm{x}$ & $\mathrm{x}$ & $\mathrm{x}$ & $\mathrm{x}$ & $\mathrm{x}$ & $\mathrm{x}$ \\
\hline Neoplasias & $\mathrm{x}$ & - & - & - & $\mathrm{x}$ & - & - & - & $\mathrm{x}$ & $\mathrm{x}$ & $\mathrm{x}$ \\
\hline Enfermagem & - & - & - & - & $\mathrm{x}$ & - & - & - & - & $\mathrm{x}$ & - \\
\hline
\end{tabular}

Fonte: Autores (2021).

Conforme os artigos 2,3 e 6, portanto, a imunoterapia é o uso de drogas produzidas por proteínas naturais que estimular o sistema imunológico humano para reconhecer e destruir mais eficazes as células cancerosas. O sistema imunológico possui células defensivas que podem proteger nosso corpo de fatores externos, ou seja, substâncias não naturais em nosso corpo, de modo que pode prevenir a poluição de qualquer substância ou o desenvolvimento de qualquer doença. Portanto, a imunoterapia é o uso de drogas produzidas por proteínas naturais que estimulam o sistema imunológico humano a reconhecer e destruir de forma mais eficaz as células cancerosas. Ao suprimir os pontos de controle do sistema imunológico, a imunoterapia aumentará ou "estimulará" a resposta antitumoral para matar as células cancerosas, em vez de agir diretamente sobre as células cancerosas e quimioterapia e terapias direcionadas (Antonia, 2014; Amador et al, 2010; Borghaei et al, 2009; Silva, 2003; Voena \& Chiarle, 2016).

Os artigos 2,3,6,7 e 8, no entanto, alguns medicamentos desenvolvidos por meio dessas proteínas podem inibir os pontos de verificação, aumentando assim a probabilidade de que as células cancerosas sejam reconhecidas e atacadas pelo sistema imunológico relatam que a descoberta do mecanismo de regulação do sistema imunológico, no qual se descobriu que os linfócitos T possuem proteínas "checkpoint" na superfície para evitar que sejam atacados pelas células de defesa do corpo. As células do melanoma usam o mesmo sistema e, portanto, são "invisíveis" para o sistema imunológico natural do corpo (Antonia, 2014; Borghaei et al ,2009; Couzin-Frankel, 2013; Immunotherapy, 2018; Parkin, 2010).

De acordo com os artigos 6,9,10 e 11, devido a essa transformação iminente, sob a orientação de imunossupressores de checkpoint e terapias direcionadas, os profissionais de uma equipe multidisciplinar têm se familiarizado gradualmente com os efeitos adversos da imunoterapia (EAI), com ênfase particular na prevenção e tratamento. A imunoterapia está prestes a mudar a prática da oncologia, mas pode-se esperar que, devido à sua natureza tóxica, vá promover novas reações adversas ou alterar as reações adversas conhecidas (Borghaei et al, 2009; Mittal et al, 2014 \& Voena \& Chiarle, 2016;)

Segundo os artigos 10 e 11, de acordo com a pesquisa de Bayer et al., provou que os resultados acima são razoáveis ${ }^{21}$. Descrever a capacidade das células cancerosas de evoluir e disfarçar o sistema imunológico, tornando-o ineficaz e criando um microambiente tumoral que pode atuar como agentes imunossupressores. Desde 1980, o Dr. Coley, um cirurgião de Nova York, conduz pesquisas sobre o papel do sistema imunológico na luta contra o câncer. Está provado que, porque a imunoterapia preenche a lacuna no campo do tratamento do câncer, a imunoterapia está ganhando cada vez mais atenção no campo científico. Naquela época, havia apenas uma opção de tratamento que constituía uma opção cirúrgica. Programa. No relatório, um paciente com infecção de erisipela no pescoço reagia a certos tipos de câncer, e foi observado que, quando ocorrem essas infecções, o volume do tumor diminui. Ele se sentia desconfortável com a necessidade de tratamento contra o câncer (Mellman et al, 2011).

Os artigos 2,3,6,7,8,9,10 e 11, mostram claramente que, com base no acima, pode ser determinado que os mecanismos fisiopatológicos da imunoterapia são diferentes e fornecem o potencial para o tratamento eficiente de uma variedade de cânceres. Também deve ser entendido que, diante da toxicidade, é necessário avaliar, gerenciar e fornecer cuidados centrados no paciente 
eficazes, seguros e eficazes. A imunoterapia se consolida como um tratamento menos agressivo e mais específico para todo tipo de câncer e pacientes com câncer (Antonia, 2014; Bayer et al, 2016; Couzin-Frankel, 2016; Immunotherapy, 2018; Silva, 2003).

Conforme o artigo 6 desde 2006, os tipos de imunoterapia são baseados em anticorpos monoclonais, transferência de células adotivas, imunossupressores de checkpoint (ICI), vacinas e outras imunoterapias não específicas. Nesse caso, o ICI se destaca, mesmo na fase tardia, o ICI também altera significativamente o curso natural da doença tumoral. Estudos clínicos demonstraram que a imunoterapia tem um potencial revolucionário. Ela pode tratar muitos tipos de câncer, regulando o sistema imunológico para promover respostas antitumorais ${ }^{1}$. Apesar do progresso, o tratamento tem sido associado a efeitos adversos da imunoterapia, que podem afetar vários sistemas de órgãos (Mellman et al, 2014; Voena \& Chiarle, 2016).

Conforme o tempo para a imunoterapia produzir reações adversas também é variável e está relacionado ao tipo de medicamento, ao plano de tratamento e ao próprio efeito, pois alguns são agudos e outros tardios. Também deve ser observado que a gravidade dessas reações adversas da imunoterapia varia, a maioria das quais são leves a moderadas, reversíveis e clinicamente controláveis (Voena \& Chiarle, 2016). Por sua vez, outros medicamentos por causa de sua letalidade, imunossupressores e substitutos são necessários (Mittal et al, 2014; Stumm, 2008).

Segundo os artigos 7 e 8 , a imunoterapia está prestes a mudar a prática da oncologia, mas espera-se que, por sua natureza tóxica, vá promover novas reações adversas ou alterar reações adversas conhecidas (McNutt, 2013; Parkhill, 2008). Como esse tipo de mudança liderada por imunossupressores de checkpoint e terapias direcionadas está no horizonte, os profissionais de uma equipe multidisciplinar devem estar familiarizados com os efeitos adversos da imunoterapia (EAI), com atenção especial à prevenção e ao tratamento (Collins et al, 2017; Sainsbury et al, 1999). As reações adversas desses dois tipos de medicamentos podem afetar a pele e as mucosas, o trato gastrointestinal, o fígado, o sistema neuroendócrino e apresentar uma ampla gama de manifestações clínicas. As reações adversas mais comuns da imunoterapia são erupção maculopapular, reações orais ou semelhantes a líquen cutâneo, úlceras orais, vitiligo, psoríase, doenças cutâneas autoimunes (Keefe \& Gibson, 2007; Sibaud, 2018). Para a imunoterapia, o principal tipo de imunoterapia disponível são os anticorpos monoclonais. Inibidores do ponto de verificação imunológico; vacinas e outras imunoterapias não específicas que geralmente estimulam o sistema imunológico. Dentre as reações adversas da imunomedicina, a toxicidade cutânea e oral (devido ao impacto significativo no paciente) também são motivos para a interrupção da terapia antineoplásica (Curry et al 2017; Stumm et al, 2008).

De acordo os artigos 9,10 e 11, o uso da imunoterapia para tratar o câncer é uma abordagem em desenvolvimento, e há alguns pontos importantes a serem revisados sobre o papel potencial do tratamento em diferentes tipos de tumores. A combinação ou sequência dessas terapias é muito ampla, sendo necessária a existência de múltiplas vias de proteção, diferentes das terapias existentes, para determinar o cronograma ideal. Também deve ser usado em combinação com imunoterapia na dose correta e combinado com radioterapia, quimioterapia ou medicamentos com outros mecanismos de ação. A vantagem do ambiente científico é que pode ampliar o conhecimento no campo do tratamento imunoterápico, sendo o uso dessa terapia pelo paciente o efeito mais específico que ela traz, podendo reduzir o tratamento mais ativo das células (Amador et al, 2010; Bayer et al, 2016; Coates \& Brest, 1999; Parker et al 2013; Parkhill, 2008).

De acordo o artigo 5 uma grande quantidade de despesas relacionadas a novos tratamentos de câncer está sendo discutida em todo o mundo, especialmente despesas relacionadas a medicamentos anticâncer. A imunoterapia parece ser adequada para a maioria dos pacientes com câncer, e algumas novas terapias direcionadas podem custar a cada paciente centenas de milhões de dólares a cada ano (Stumm et al, 2008).

Outro diferencial é a forma como os pesquisadores fazem a mudança no gene das células T: em vez de utilizar um vírus para infectar a célula do sistema imune e promover a mudança genética, técnica mais amplamente utilizada, são empregados fragmentos não infecciosos de DNA. Esse é um dos primeiros grupos fora da Europa e Estados Unidos a conseguir levar o gene CAR para o núcleo da célula sem usar o vírus. E isso tem uma série de vantagens potenciais que estamos explorando, entre elas, 
a redução no custo os resultados da etapa da pesquisa em animais foram recentemente publicados na revista Human Gene Therapy o grupo se prepara para a etapa com pacientes. Além do Inca, o trabalho é conduzido em parceria com a Universidade Estadual de Campinas (Unicamp), o Centro Infantil Boldrini e a Fundação Oswaldo Cruz (Durkee et al, 2016).

Conforme o artigo 10, os enfermeiros que atuam no setor hospitalar, principalmente aqueles que prestam atendimento especializado ao paciente oncológico, devem estar aptos a cuidar de todos os pacientes oncológicos com métodos que garantam sua integridade, para que as ações de enfermagem envolvidas no cuidado ao paciente oncológico sejam participativas no diversos níveis de atuação Além da resolução de conhecimentos técnico-científicos, os profissionais também devem possuir habilidades interpessoais, que favoreçam as ações de saúde e as práticas educativas, a fim de prevenir, detectar precocemente o câncer e contribuir para o tratamento do câncer (Freire, 2019).

O enfermeiro que ainda está nesta faixa deve ser capaz de avaliar o uso da tecnologia em termos de segurança, eficácia, custo-efetividade, impacto social, etc., e focar nos aspectos éticos envolvidos nas diferentes situações, para que possa se beneficiar pacientes em qualquer forma de decisão (Secoli et al 2005).

Através da análise do mercado de trabalho e das diferentes áreas de atuação do enfermeiro, a competência profissional é considerada um requisito básico, o que obriga o enfermeiro a desenvolver um pensamento crítico e reflexivo para revelar a realidade e propor ações transformadoras para os indivíduos, a fim de prestar uma ajuda de qualidade. bem como clientes e A satisfação dos membros da família é o objetivo. Levando em consideração essas considerações, destacamos que o enfermeiro que atua no serviço de oncologia precisa ter uma imagem pessoal e profissional para que possa efetivamente desenvolver suas funções, aliando conhecimento técnico-científico, enfermagem humanizada e personalizada (Backer et al, 2008; Ferreira \& Kurcgant, 2009).

O manejo dos eventos adversos imunorrelacionados (IRAEs) está em aprimoramento juntamente com a experiência clínica do profissional da enfermagem faz toda a diferença nos protocolos dos IRAEs mais comuns como rash cutânea o enfermeiro tem um papel fundamental (Institute, 2010). Até o momento não há estudos clínicos prospectivos sobre a avaliação e manejo de IRAE. Porém, atualmente as diretrizes de gerenciamento dos eventos adversos empregadas descrevem o uso de terapias imunossupressoras empiricamente, baseadas na experiência clínica do uso desses agentes em estudos clínicos, além de considerar o que já é conhecido em relação aos seus potenciais mecanismos de toxicidade (Postow, 2015). O manejo geral dos IRAEs é baseado em um método de retenção da droga isolada, em casos leves; início de medicação esteroide oral e consulta especializada para casos moderados; e adição de imunossupressores com antagonista alfa do fator de necrose tumoral (TNF- $\alpha$ ), inibidor de calcineurina ou outros agentes, junto com medidas de suporte, conforme requerido ${ }^{42}$. Acompanhamento e vigilância clínica dos sinais e sintomas dessas toxicidades ao longo da terapia são de extrema importância. Os IRAEs podem desenvolver diferentes características ao longo do tratamento, além de poder ocorrer após o término da terapia (Postow, 2015; Squibb, 2016).

Eventos adversos imunorrelacionados para graduar a toxicidade de um evento adverso utiliza-se o guia internacional: Terminologia Comum dos Eventos Adversos (CTCAE). Acompanhamento e vigilância clínica dos sinais e sintomas dessas toxicidades ao longo da terapia são de extrema importância. Os IRAEs podem desenvolver diferentes características ao longo do tratamento, além de poder ocorrer após o término da terapia. Tanto o manejo dos IRAEs mais comuns como rash cutânea e colite, quanto os menos frequentes como pneumonite, são de interesse médico e precisam ser detectados o quanto antes para que a condução desses eventos seja efetiva (Naidoo et al, 2015.

As toxicidades mais comuns com agentes que não sejam inibidores anti-CTLA-4 e PD1/PD-L1 incluem fadiga, reações de infusão, febre, eritema local e desconforto no local da aplicação. Estas toxicidades podem ser manejadas com pré-medicação como: acetaminofeno e um anti-histamínico, bem como a redução na taxa de infusão (Postow, 2015). 
O manejo dos IRAEs é baseado em relatórios de segurança das indústrias farmacêuticas, opinião de especialistas e no conhecimento das doenças autoimunes (Squibb, 2016). Muitos destes eventos adversos são sensíveis a esteroides e tem resolução dentro de 6 a 12 semanas (Kronbichler et al, 2015).

A perspectiva da equipe de enfermagem sobre a relação entre acreditação e segurança do paciente se refere à pontuação clara de meios/estratégias de cuidado mais seguro advindos do processo de acreditação, em especial por aspectos de teor instrumental e sistemático embutidos no processo de trabalho. Pontuam, ainda, a segurança do paciente ora como dependente (por meio dos momentos transversais de manutenção do selo de qualidade), ora como independente da acreditação, já que o cuidado seguro transpõe (é “maior”) que a certificação em si. Nesse contexto, revelou-se valorização do processo de acreditação rumo à assistência mais segura, mas, também, criticidade entre os trabalhadores de enfermagem (Oliveira, 2020).

\section{Conclusão}

A imunoterapia está voltada para tratamentos de câncer com um modelo em desenvolvimento, existem alguns pontos que precisam ser revistos em relação ao potencial de tratamento em diferentes tipos de tumores. Devido suas combinações ou sequência referente aos métodos de tratamento são muito amplos, sendo necessário ter uma variedade de métodos de proteção diferentes dos métodos de tratamento existentes e determinar o calendário ideal. Esses avanços representam a compreensão das mutações que promovem o desenvolvimento do câncer, a transdução de sinal que leva à progressão do tumor e os avanços na compreensão da interação entre as células tumorais e o sistema imunológico estão revolucionando o tratamento do câncer.

Esse é, sem dúvida, um importante fator limitante da comercialização desses medicamentos no Brasil. No Brasil, alguns bons tratamentos de câncer ainda precisam ser registrados para enfatizar a lacuna entre os tratamentos usados aqui e os usados em países desenvolvidos. Em comparação com os registros efetivamente disponíveis no SUS, a diferença significativa entre o que é registrado e o que é utilizado em um sistema de saúde privado é tão importante. A menos que os preços dos medicamentos se tornem mais razoáveis no futuro próximo, e a avaliação das tecnologias em saúde no SUS seja orientada por padrões reconhecidos e limites de custo-efetividade predeterminados, essas novas terapias anticâncer irão impor restrições aos países em desenvolvimento. O Brasil como exemplo, a diferença entre a prática internacional e a prática do meu país tende a aumentar muito (INCA, 2015; Stiefel, 2006).

Com isso os estudos e a velocidade com que novos tratamentos são aprovados e o alto custo das medicações dificultam a disponibilização de terapêuticas em países como o Brasil. Desta forma apresentamos nesta revisão, esses novos tratamentos e comparamos sua disponibilidade nos sistemas público e privado de saúde no Brasil com os países desenvolvidos. Além disso, salienta-se que tais terapias ainda não são disponíveis no SUS por se tratar de um tratamento de alto custo, encontrando-se limitadas no Brasil devido atrasos nas liberações da ANVISA.

Experiência profissional e qualificações são deve a ser reconsiderado no contexto da atual contratação de profissionais de enfermagem na atuação na área de oncologia. Compreendemos as limitações de pesquisas desta natureza, mas enfatizamos que a pesquisa deve despertar reflexões de gestores, centros de treinamento e o futuro. Faz-se necessário o enfermeiro estar capacitado para realizar o cuidado adequado para seus pacientes. Realizando a capacitação dos enfermeiros sobre a utilização do tratamento com imunoterapia, e orientado sobre os principais exames e avaliar para ser realizado o tratamento de imunoterapia; orientar sobre as reações adversas da imunoterapia. Outras pesquisas devem serem para determinar a estratégia de gestão da unidade de câncer de acordo com as qualificações dos enfermeiros.

Por fim, sugere-se que a imunoterapia por estar em evolução no Brasil, são destinadas a controlar o sistema imunológico do próprio paciente. O potencial deste método para melhorar a sobrevivência a longo prazo em vários tipos de tumores está sendo 
estudado atualmente. Agora, é importante determinar como usar a imunoterapia de forma mais eficaz para obter o melhor resultado possível, trazendo uma qualidade para esse paciente em tratamento.

\section{Referências}

Amador, D. D., Gomes, I. P., Coutinho, S. E. D., Costa, T. N. A. \& Collet, N. (2010). A vivência do cuidado em oncologia pediátrica e a busca pela produção do conhecimento. Rev enferm. UFPE on line; 4 (2): 666-72. http://www.revista.ufpe.br/revistaenfermagem/index.php/revista/article/view/851.

Antonia, S., James. J. L. \& Ascierto, P. A. (2014). Immunooncology combinations: a review of clinical experience and future prospects. Clinical Cancer Research. 20 (24), 6258-6268.

Backes, V. M. S., Lino, M. M., Prado, M. L. do., Reibnitz, K. S. \& Canaver, B. P. (2008). Competência dos enfermeiros na atuação como educador em saúde. Rev Bras Enferm. 61(6), 858-65. http://www.scielo.br/pdf/reben/v61n6/a11v61n6.pdf..

Bayer, V., Amaya, B., Baniewicz, D., Callahan, C., Marsh, L. \& McCoy, A. S. (2016). Cancer Immunotherapy: An evidencebased overview and implications for practice. Clinical journal of oncology nursing. 2, 21.

Borghaei, H., Smith, M. R. \& Campbell, K. S. (2009). Immunotherapy of cancer. European Journal of Pharmacology. 625, 41-54.

Bray, F. et al. (2018). Global cancer statistics estimates of incidence and mortality worldwide for 36 cancers in 185 countries. CA: a cancer journal for clinicians, Hoboken; 68 (6) 394-424.

Coates, A. S. (1999). Breast cancer: delays, dilemmas, and delusions. Lancet, 353(9158), 1112-3.

Collins, L. K., Chapman, M. S., Carter, J. B. \& Samie, F. H. (2017). Cutaneous adverse effects of the immune checkpoint inhibitors. Current Problems in Cancer. 41(2), 125-28.

Couzin-Frankel, J. (2013). Cancer immunotherapy. Science, vol. 342, Issue 6165, p. 1417.

Curry, J. L, Tetzlaff, M. T., Nagarajan, P., Drucker. C., Diab, A. \& Hymes, S. R. (2017). Diverse types of dermatologic toxicities from immune checkpoint blockade therapy. J Cutan Pathol. 44, 158-76.

Durkee, B. Y., Qian, Y., Pollom, E. L., King, M. T., Dudley, A. S. \& Shaffer, J. L, et al. (2016). Cost Effectiveness of Pertuzumab in Human Epidermal Growth Factor Receptor 2-Positive Metastatic Breast Cancer. J Clin Oncol. 34(9), 902-9.

Ferreira, J. C. O. A. \& Kurcgant, P. (2009). Capacitação profissional do enfermeiro de um complexo hospitalar de ensino na visão de seus gestores. Acta Paul Enferm. 22(1),31-6. http://www.scielo.br/pdf/ape/v22n1/a05v22n1.pdf 7.

Freire, D. (2019). Imunoterapia: a virada do sistema imunológico contra o câncer. Cienc. Cult. vol.71 no.4 São Paulo. /10.21800/2317-66602019000400006. Hanahan, D. \& Weinberg, R.A. (2000). The hallmarks of cancer. Cell. 100 (1), 57-70. https://doi.org/10.1016/S0092-8674(00)81683-9.

Immunotherapy for Melanoma Skin Cancer. (2018). American Cancer Society. https://www.cancer.org/cancer/melanomaskincancer/treating/immunotherapy.html.

Institute, N. C. (2010). Common Terminology Criteria for Adverse Events (CTCAE): Version 4.032010. http://evs.nci.nih.gov/ftp1/CTCAE/CTCAE_4.03_201006 14_QuickReference_8.5x11.pdf.

Instituto Nacional de Câncer (2015). Coordenação de Prevenção e Vigilância: Estimativa 2016: Incidência de câncer no Brasil. Instituto Nacional de Câncer José Alencar Gomes da Silva - Rio de Janeiro: INCA.

Keefe, D. M. . \& Gibson, R. J. (2007). Mucosal injury from targeted anti-cancer therapy. Supportive Care Cancer. 15, 483-90.

Knechtel, Maria do Rosário. Metodologia da pesquisa em educação: uma abordagem teórico-prática dialogada. Curitiba: Intersaberes, 2014.

Kronbichler, A. et al. (2015). Frequency, risk factors and prophylaxis of infection in ANCA - associated vasculitis. Euro Journal Clinical Invest. 346 - 368.

Mathers, et al. (2013). Anticancer Therapy. J Pharmacovigilance. 1 (4), 1-4.

McNutt, M. (2013). Cancer immunotherapy. Science. 342, (6165), 1417.

Mellman, I., Coukos, G. \& Dranoff, G. (2011). Cancer immunotherapy comes on age. Nature. 480, 480-9.

Mendes, K. D. S., Silveira, R. C. C. P. \& Galvão, C. M. (2008). Revisão integrativa: Método de pesquisa para a incorporação de evidências na saúde e na enfermagem. Texto Contexto Enferm, Florianópolis. 17(4), 758-64.

Mittal, D., Gubin, M. M., Schreiber, R. D. \& Smyth, M. J. (2014). New insights into câncer immunoediting and its three componente phase - elimination, equilibrium and escape. Current opinion in immunology. 27, 16-25.

Mittal, D., Gubin, M. M., Schreiber, R. D., Smyth, M. J. (2014). New insights into cancer immunoediting and its three componente phase - elimination, equilibrium and escape. Current opinion in immunology. 27,16-25.

Naidoo, J. et al. (2015). Toxicities of the anti-PD-1and anti-PD-L1 immune checkpoint antibodies. Ann. Oncol. 26,2375 - 2391. 
Research, Society and Development, v. 10, n. 6, e38710615902, 2021

(CC BY 4.0) | ISSN 2525-3409 | DOI: http://dx.doi.org/10.33448/rsd-v10i6.15902

Oliveira, J. L. C. de. Et al. (2009). Interface entre acreditação e segurança do paciente: perspectivas da equipe de enfermagem. Ver.Esc. Enferm. USP, 54:e 03604. /10.1590/S1980-220X2018053703604.

Oncologia. (2021). O que é imunoterapia? http://www.oncoguia.org.br/conteudo/o-que-e-imunoterapia/7957/922/.

Parker, C., et al. (2013). Alpha emitter radium-223 and survival in metastatic prostate cancer. ALSYMPCA Investigators. $N$ Engl J Med. 369 (3), $213-23$.

Parkhill, A. L. (2013). Oral Mucositis and Stomatitis Associated with Conventional and Targeted Anticancer Therapy. J Pharmacovigilance. 1,4,1-4.

Parkin, D. M., Boyd, L., \& Walker, L. C. (2010). The fraction of cancer attributable to lifestyle and environmental factors in the UK in 2010. Br J Cancer. 105 (2), 77-81.

Peterson, D. E., O’Shaughnessy, J. A., Rugo, H. S, Elad S, Schubert, M. M., \& Viet, C. T et al. (2016). Oral mucosal injury caused by mammalian target of rapamycin inhibitors: emerging perspectives on pathobiology and impact on clinical practice. Cancer Medicine. 5 (8), $1897-1907$.

Postow, M. (2015). Managing immune checkpoint-blocking antibody side effects. American Society of Clinical Oncology education book/ ASCO American Society of Clinical Oncology Meeting, pg. $76-83$.

Rosenberg, S. A. \& Restifo, N. P. (2015). Adoptive cell transfer as personalized immunotherapy for human cancer. Science. 348(6230), 62-8.

Sainsbury, r., Johnston, c. \& Haward, B. (1999). Effect on survival of delays in referral of patients with breast-cancer symptoms: a retrospective analysis. Lancet., London, 353, 1132-1135.

Secoli, S. R., Padilha, K. G. \& Leite, R. C. B. O. (2005). Avanços tecnológicos em oncologia: reflexões para a prática de enfermagem. Revista Brasileira de Cancerologia Enfermería Global No 37 Abril 2015 Página 322;51(4), 331-337. http://www.inca.gov.br/rbc/n_51/v04/pdf/revisao4. pdf.

Sibaud, V. (2018). Dermatologic Reactions to Immune Checkpoint Inhibitors: Skin Toxicities and Immunotherapy. American of Journal Clinical Dermatology. $19(3), 345-361$

Silva, W. D. \& Mota, I. B. (2003). Imunologia básica e aplicada. (5a ed.), Guanabara Koogan, 2003.

Souza, M. T., Silva, M. D. \& Carvalho, R. (2010). Revisão integrativa: O que é e como fazer. Einstein. 8 (1), 102-6.

Squibb, B. (2016). Opdivo (nivolumab): Immune-Mediated Adverse Reactions Management http://www.opdivohcp.bmscustomerconnect.com/servelet/servelet.FileDownload?file=00Pi00000Hj19REAR.

Stiefel, F. (2006). Understanding why women delay in seeking help for breast cancer symptoms. Letter to the Editor. Journal of Psychosomatic Research. Oxford, 60, 309- 310 .

Stumm, E. M. F., Leite, M. T. \& Maschio, G. (2008). Vivências de uma equipe de enfermagem no cuidado a pacientes com câncer. Cogitare Enferm. 13 (1), 75-82. $/ 10.5380 /$ ce.v13i1.11955.

Voena, C. \& Chiarle, R. (2016). Advances in cancer immunology and cancer immunotherapy. Discovery medicine. 21, 125-33.

Yaddanapudi, K., Mitchell, R. A. \& Eaton, J. W. (2013). Cancer vaccines: Looking to the future. Oncoimmunology. 2 (3), 23403. 